\title{
REVIEW
}

\section{Identifying and Targeting the Cause of Cancer is Needed to Cure Cancer}

Adouda Adjiri

To view enhanced content go to www.oncologytherapy-open.com

Received: November 23, 2015 / Published online: January 20, 2016

(c) The Author(s) 2016. This article is published with open access at Springerlink.com

\section{ABSTRACT}

Cancer cells continue to challenge scientists and oncologists due to the phenomenon of resistance. Moreover, recurrence, as seen in many treated patients, shows that currently-used anti-cancer drugs are unable to prevent the development of new cancer cells harboring new mutations. The purpose of this paper is to try to answer some of the questions regarding why cancer arises and why evolution would naturally lead to the development of cancer. Providing answers to these questions may shed new light on cancer development and potential causes of cancer. This work demonstrates that (1) cancer hallmarks are a series of events that can be organized in three consecutive stages; (2) cancer may develop when cells seek immortality; (3) heterogeneity in tumors may be explained by cancer cells not following universal laws for division; (4)

A. Adjiri (夰)

Physics Department, Faculty of Sciences, Sétif-1

University, 19000 Sétif, Algeria

e-mail: adouda.adjiri@yahoo.com;

adouda.adjiri@outlook.com evolution may not have selected for cancer; (5) currently-used anti-cancer drugs, with telomerase and poly adenosine diphosphate ribose polymerase inhibition given as examples, show that we may not be on the right track, as these drugs are probably targeting molecular symptoms of tumors but not their cause; and (6) after an attempt to define the cause of cancer, the potentials of immunotherapy are discussed. Future anti-cancer drugs should be able to shrink the original tumor(s) and most importantly prevent the rise of new cancer cells in treated patients. In order to achieve this goal, new drugs must target the cause of cancer. Therefore, future research must focus on identifying potential causes of cancer common to all types of cancers. Finally, while immunotherapy holds great prospects for future cancer cure and prevention, global action is needed to reduce harmful substances known to contribute to the development of cancer in the environment.

Keywords: Apoptosis; Cancer-cell resistance; Cancer hallmarks; Cause of cancer; Evolution; Immortality; Senescence 


\section{INTRODUCTION}

Cancer is the most prominent and challenging disease in humans today despite the great efforts employed for prevention and the cure achieved in a significant proportion of cancers by surgery, radiotherapy or chemotherapy, especially when detected early. Huge amounts of resources have been devoted to cancer research but clinicians and oncologists are still struggling as currently used therapeutic strategies are not entirely effective, and we are far from erasing this disease that continues to kill thousands worldwide every year.

Radiotherapy is one of the main modalities of cancer treatment as it has an excellent control over the tumor with the preservation of normal tissues and less systemic influences. However, tumors treated with radiation recur in some cases with radio-resistance, leading eventually to treatment failure [1]. In recent years, radiation therapy technologies have known an important improvement and development [2]; however, the most innovative strategy has failed to achieve a complete remission, and patients often suffer from tumor recurrence and/or distant metastasis after radiation therapy [3]. Moreover, radiation is shown to induce genetic instability and chromosomal rearrangements, and many of these rearrangements are similar to those found in human cancers [4]. It has also been shown that a sub-lethal dose of photon irradiation promotes cancer cell metastasis by increasing their migration and invasion potential [5].

More recently, hadron therapy emerged as an alternative way of treating cancer and consists of irradiating tumors with protons (proton therapy) or light nuclei (alphas, carbon ions). Compared to standard radiotherapy, it presents two major advantages: a precise ballistics, with a finite range and a maximum dose deposition at the end of the path of the ions (i.e. Bragg peak) and an improved biological efficiency in the Bragg peak area. This allows to better target the tumor while the surrounding healthy tissues are preserved [6]. Hadron therapy looks, therefore, more promising, mainly in tackling radio-resistant tumors; however, studies are needed to assess the long-term effects in terms of recurrence and/or other symptoms.

Chemotherapy is mainly based on cytotoxic drugs which often result in severe side effects and poor quality of life. The number of drugs used against cancer is increasing, targeting a myriad of molecules proven to play a role in the development, invasion and/or metastasis, to just discover down the road that their curative effect is not as effective as predicted. This has pushed scientists and oncologists to develop individualized therapies based on the genetic profile of individual cancers [7]. On a practical level, however, it may not be easy to devise personalized treatments for that many different cancers in that many different patients, knowing the changing character of cancer cells. Moreover, the cost of such a treatment strategy could also be problematic.

Surgery, on the other hand, is effective in tumors before they reach the stage of invasion or metastasis, but surgery cannot do much once the tumor has spread to other parts of the body. Confronted with a frustrating situation raised by chemo and/or radiation resistance, clinicians often combine different modalities in adjuvant therapies. Combined radio-chemotherapy protocols are used in many cancers, such as glioblastoma multiforme or pancreas cancer [8]. Particle therapy in combination with 
immunotherapy is equally used as another modality in cancer treatment [9].

This gloomy image of cancer therapy tells that a cure has not been achieved in cancer even though many successful stories of complete remission have been described in literature. One reason for this is the complexity of the disease involving a multitude of changes at the epigenetic and genetic levels, not overlooking the influence of the diverse environmental factors affecting these changes, adding another layer of complexity to the problem of tumor genesis. The second reason is our limited understanding of the evolutionary dynamics that govern the process of tumor formation which led us to cancer treatment strategies that are not very effective.

This paper tries to answer two important questions which have not been asked before; why cancer develops and why evolution would select for cancer. For this, we first need to review cancer hallmarks through a new window in order to shed light on the sequence of the events leading to tumor formation. Moreover, heterogeneity within a tumor is examined here under the lenses of evolution. Furthermore, this work discusses two types of molecules targeted by currently used anti-cancer drugs; the telomerase and poly (ADP-ribose) polymerase (PARP) proteins. Resistance to these and other non-cited anti-cancer drugs raises attention to the cause of cancer. After an attempt to define the cause of cancer, after an attempt to define the cause of cancer, the potentials of immunotherapy are discussed.

This article is based on previously conducted studies and does not involve any new studies of human or animal subjects performed by any of the authors.

\section{CANCER HALLMARKS ARE A SERIES OF EVENTS THAT CAN BE ORGANIZED IN THREE CONSECUTIVE STAGES}

Cancer is thought of as a genetic disease known to result from a series of molecular events that fundamentally alter the normal properties of cells, leading to uncontrolled cell division [10]. Malignant tumors have a monoclonal character, meaning that each tumor arises from a single transformed cell [11]. The development of a malignant tumor from a normal cell usually occurs over a long period of time. Thus, multiple steps are required for cancer to arise, as shown in laboratory animal models of cancer development, providing clear evidence that specific stages in malignant transformation can occur discretely [12].

Cancer arises through the sequential accumulation of mutations in oncogenes and tumor suppressor genes. The mathematical studies of Nordling [13] suggested that six or seven sequential mutations are required. However, recent studies have shown that only three sequential mutations are required to develop cancer, as demonstrated in lung and colon adenocarcinomas [14].

Hanahan and Weinberg have outlined the key biological properties, or hallmarks of cancer cells, which include: having self-sufficiency in growth signals, loss of sensitivity to anti-growth signals, loss of sensitivity to programmed cell death or apoptosis, immortality, altered metabolic properties, higher mutation rates and deregulated cellular energetics, avoiding immune destruction, ability to attract blood vessels, and the capacity to invade tissues and become metastatic [15]. 
These cancer hallmarks could be seen as characteristics earned after the birth of the first cancerous cell. This first cancerous cell came to be formed after having accumulated a number of epigenetic and/or genetic changes through a high rate of mutation, making it earn, among other characteristics, self-sufficiency in regard to growth signals and loss of sensitivity to anti-growth signals. The survival and division of this first cancerous cell means failure of apoptosis to kill such a transformed cell while inferring failure of senescence as well.

Among the important changes occurring in the cell in order to become cancerous are those affecting its telomeres as these molecular complexes play crucial roles in the life of any cell still in the phase of growth and division. A cancerous cell keeps its telomeres in shape through reactivated telomerase or an alternative telomere lengthening (ATL) mechanism, in a phenomenon commonly described as immortalization. The reacquisition of a telomerase or the development of an ATL mechanism may not be solely for immortalization purposes per se but could also play a role in setting different gene-regulation patterns. Moreover, it has been shown that telomere-associated proteins have extra-telomeric roles in regulating diverse signaling pathways in mammals [16].

Altered metabolic properties and deregulated cellular energetic are easily seen in tumors; a property particularly explored in some medical imaging techniques [17]. And when glucose metabolism is concerned, cancer cells show a strong avidity for this nutrient [18]. This avidity for glucose may signify that cancer cells need more energy in order to function independently as a tumor mass separated from normal and regulated tissue cells. Moreover, the ability to attract blood and/or lymph vessels comes once a tumor is formed but not before. In this regard, targeting angiogenesis can result in the shrinking of the tumor but may not prevent the rising of new cancerous cells. Resistance to anti-angiogenic treatment drugs has been reported and multiple mechanisms have been proposed in the development of tumor resistance to anti-angiogenic therapy [19].

Advancing further in the developmental stages of cancer, local invasion takes place followed by metastasis as the final stage. And for metastases to arise, cancerous cells have to travel through blood or lymph vessels in order to reach farther territories. Cancer cells have, thus, to overcome the immune system in all its capacities. This escape could be explained by the fact that our immune cells are unable to see cancer cells in a manner similar to how they see invading bacterial cells.

Colotta et al. [20], in their paper in Carcinogenesis, referred to inflammation as the seventh hallmark of cancer. Inflammation also causes resistance to apoptosis, secretion of pro-angiogenic and immunosuppressive factors, fostering invasion and metastasis [21]. Inflammation might be a sign of an ongoing crisis after which some cells may escape death and become immortal.

Summarizing cancer hallmarks can be reduced to three major stages in the following order: (1) reprogramming cell growth; (2) birth and survival of the first formed cancerous cell; and (3) proliferation, invasion and metastasis. Stage (1) harbors discreet epigenetic and/or genetic changes which lead to immortality. In stage (2), the successful implantation of this reprogramming gives birth to the first cancerous cell, the survival of which requires resistance to apoptosis and/or senescence. Stage (3) assures further division of this cancerous cell that had acquired self-sufficiency towards growth signals and had lost sensitivity to anti-growth signals. Thus, once a malignant cell is formed and has 
survived a first round of cell-division, invasion and metastasis may follow as an inevitable consequence.

\section{CANCER ARISES WHEN CELLS SEEK IMMORTALITY}

Reviewing cancer hallmarks brings us to the first important question behind transformation, and that is why some cells go cancerous. Understanding the why before the how could open a new window on cancer development that may lead us to new and better treatment strategies.

Life cycles in higher eukaryotes, including humans, are characterized with distinct stages going from embryogenesis, birth, growth, maturity, senescence and aging followed inevitably by death. During these life cycles, individual cells also go through stages from immaturity to maturity, senescence and death. The hematopoietic cell cycle offers such an example when our blood cells are concerned.

In this view, and at a cellular level, senescing to eventually die might be regarded by individual cells as a stressful event. Becoming immortal could, thus, be a way of coping as tumor formation gives cells an unlimited cell-division capacity. In this case, escaping senescence and, ultimately, apoptosis would be the major hurdle to overcome in order to become immortal. And accumulated stress along this path might be behind age-related cancers.

At an individual level, however, and in the presence of a known and incessant stress, exposed cells may create cancer as a way of coping as well. In this case, escaping apoptosis and/or senescence would similarly be the hurdles to overcome in order to become immortal.

\section{Escaping Senescence}

In physiological settings, somatic cells do not divide indefinitely because of a process termed cellular senescence that is defined as a permanent cell resting phase and is related to cell aging [22]. Cells reach senescence in two different ways; (1) as a result of telomere shortening after a number of cell divisions, called replicative senescence, or (2) as a response to stress, and called stress-induced premature senescence [23]. Replicative senescence is believed to have evolved in higher eukaryotes and, particularly, in mammals, as a protective measure from developing cancer [24, 25].

Senescent cells, which arrest in the G1 phase of the cell cycle, are no longer able to synthesize DNA and replicate. This is mainly due to the inactivation of cyclin-dependent kinase (CDK) by CDK inhibitors (CDKI) [26]. The senescence pathway includes p16INK4A, CDK and RB, the expression of which is highly deregulated in nearly all human cancers [27].

In melanoma, the three susceptibility genes identified so far are those involved in the process of cellular senescence; p16INK4A, ADP-ribosylation factor (ARF) and CDK4 [28]. The locus harboring the first two genes encodes two distinct protein products that start from different first exons but share some coding sequence in two different reading frames. Both proteins are suppressors of cell proliferation, and both are established as important tumor suppressors in humans, being mutated in a substantial range of sporadic tumor types [29]. The CDK4 encodes the kinase that is inhibited by p16. The RB pathway appears critically important in the suppression of melanoma development, since nearly all human melanoma cell lines tested show disruption of this pathway, via p16 or RB1 deficiency, CDK4 mutation or over-expression of cyclin D1 [30, 31]. 
Moreover, it has been reported that tumorigenic cells spontaneously emerged from senescent cells [32]. We know that senescent cells differ from pre-senescent cells since once arrested cannot be stimulated to re-enter the cell cycle by physiological mitogens and become resistant to apoptotic cell death while acquiring altered differentiated functions [33]. In addition, those cells that entered senescence are known to secret pro-inflammatory and pro-proliferative factors which are phenomena-associated with cancer and, thus, may cooperate in the creation of cancer.

\section{Escaping Apoptosis}

Apoptosis, defined as a programmed cell death, plays an important role in tumor suppression. Kerr et al. [34] were the first to link apoptosis to the elimination of potentially malignant cells, hyperplasia and tumor progression. Therefore, programmed cell death is thought to have evolved as a measure to maintain tissue homeostasis by removing abnormal or damaged cells, and also to remodel tissues during morphogenesis $[35,36]$.

Apoptosis can occur either through an intrinsic or an extrinsic pathway and its mechanism is well-described and characterized by a series of morphological changes leading to the formation of apoptotic bodies [37]. The primary regulators of apoptosis are proteins belonging to a group known as the Bcl-2 family. These proteins can either be pro-apoptotic or anti-apoptotic and, generally, the mechanisms by which evasion of apoptosis occurs can be summarized into: (1) disrupted balance of pro-apoptotic and anti-apoptotic proteins; (2) reduced caspase function; and (3) impaired death receptor signaling [37].

The p53 tumor suppressor is a key transcription factor regulating cellular pathways such as DNA repair, cell cycle, angiogenesis, senescence, and also apoptosis. This protein acts as an important defense mechanism against cancer onset and progression and defects in the p53 gene have been linked to more than 50\% of human cancers [38]. Moreover, some target genes of p53 involved in apoptosis and cell cycle regulation are aberrantly expressed in melanoma cells, leading to abnormal activity of p53 and contributing to the proliferation of these cells [39].

Figure 1 portrays a detour from regulated cell division. Knowing that cancerous cells are malignant, their rise could be a result of senescence evasion in old individuals resulting in different types of age-related cancers. On the other hand, evasion of apoptosis may occur regardless of age and could be a continuous process from the birth of the first cancerous cell all the way to invasion and metastasis.

In conclusion senescence and apoptosis, being two important cancer hallmarks, are the major hurdles to be overcome by cells desiring to become cancerous. Therefore, deciphering the molecular events occurring during senescence will shed light on how cells evade senescence and become resistant to apoptosis, and melanoma offers a very good model for such studies.

\section{HETEROGENEITY WITHIN A TUMOR MASS COULD BE EXPLAINED BY CANCER CELLS NOT FOLLOWING UNIVERSAL LAWS FOR THEIR DIVISION}

If immortality were the common drive in cancer development and the force behind transformation, why are cells within a tumor heterogeneous? In other words, why shouldn't there be a common pathway for cancer formation when the goal is one? 
A. The path of regulated growth through regulated cell division.

Growth -------------------->Maturity -------------->Senescence --------->Aging --------->Death

B. Cancer-causing stressors lead to apoptosis evasion and/or senescence evasion.

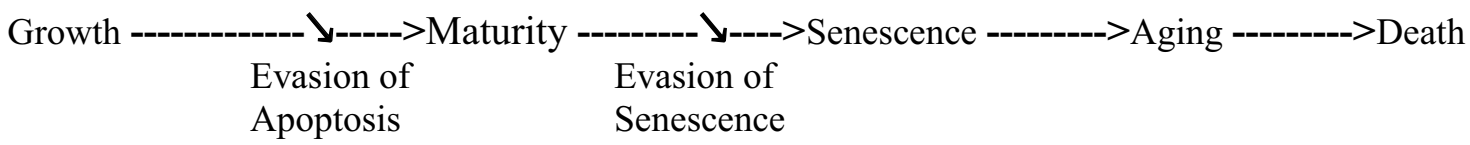

C. Present and future drug targets

Action of future anticancer drugs

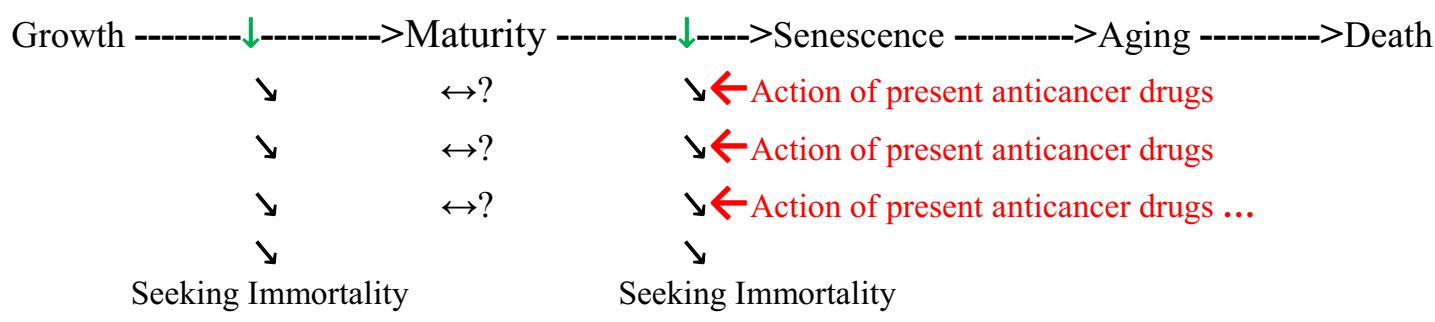

Fig. 1 Action of future anti-cancer drugs. a The path of regulated growth through cell division. b Cancer-causing stressors lead to apoptosis evasion and/or senescence evasion. c Present and future drug targets. ( $\searrow$ ): Evasion of apoptosis and/or evasion of senescence result in tumor

One remarkable characteristic of cancer cells is their high capacity for adaptation. This adaptation is shown in the ability of cancer cells to co-opt metabolic pathways. Co-option indicates that cancer cells are unable to create de novo their own pathways; assuring their metabolism and proliferation. Therefore, hijacking different metabolic pathways results in different molecular symptoms seen in different cancers. In this regard, heterogeneity seen within a tumor mass could be explained by cancer cells not following universal laws for their division. Moreover, not being subject to universal rules for cell division permits such formation as cells seek immortality. $(\leftarrow)$ : Symptomatic steps targeted with present anti-cancer drugs when cells are already off the trajectory of regulated growth. $(\downarrow)$ : upstream step to target in the design of future anti-cancer drugs

cells to mutate at a high rate, fulfilling yet another important cancer hallmark.

In physiological conditions, the universal laws for cell division assure a regulated growth, safeguarding our tissues and organs. These laws are seen in checkpoint controls during cell cycle division in eukaryotes [40]. The cell cycle is guarded at three checkpoints: at the G1/S boundary, the G2/M boundary, and the metaphase/anaphase boundary. p53 is the quintessential guardian of the genome stability [41] and mutations in this transcription factor are among the most common genetic alterations in human cancer; being mutated in 
nearly $90 \%$ of human melanomas [42]. p53 controls the cell cycle by mediating DNA damage responses to a variety of cellular stresses and inducing cell cycle arrest [43, 44] senescence [45], and apoptosis [46, 47].

In conclusion, and because metabolic pathways hijacked by cancerous cells are numerous, the symptoms are numerous, thus making it difficult to devise a single and effective anti-cancer drug or therapy. Targeting each molecule on each route followed by each type of cancer has not resulted in a cure of cancer, as seen with the resistance and recurrence phenomena. The only way out of this rut is to look for the cause of cancer and design new anti-cancer drugs targeting the cause of cancer rather than its molecular symptoms.

\section{CANCER MAY NOT HAVE BEEN SELECTED FOR BY THE NATURAL FORCES GOVERNING EVOLUTION}

Cancer is thought of as an evolutionary process involving successive generations of cells, which are progressively further advanced towards cancerous growth [11] and that cancer progression is a form of somatic evolution in which certain mutations give a cell a selective proliferation advantage [48]. Therefore, trying to see cancer development through the lenses of evolution leads to the second important question and that is why evolution would select for cancer.

Evolution might be seen as a universal law through which organisms and non-living matter evolve toward higher structures of higher complexity and function. In this regard, evolution serves life by working according to universal laws that are constructive but not destructive. Cancer development on the opposite, works against life, as it destroys tissues and obstructs organs' function. In doing this, cancer development shows a selfish character when tumors are formed, neighboring tissues invaded and distant metastasis formed. Consequently, cancer cannot be seen as following universal laws because universal laws create, preserve and evolve towards higher organization, but cancer works towards disorganization, destruction and death. Evolution appears, therefore, to be a purposeful process, whereas cancer development is, at cellular and individual levels, purposeless, as it creates anarchy and death.

If evolution builds and cancer destroys; then it may be inadequate to say that cancer was selected for by natural forces governing evolution. To select something is to choose what is most advantageous and cancer is not advantageous in the sense that cancer is a pathological event that causes suffering and death. Instead, it might be safer to say that evolution did not prevent cancer formation or hamper its appearance. But why evolution would allow cancer to appear since it is disadvantageous?

In an attempt to answer such a difficult question, it might be easier to ask instead what would have happened had evolution worked against cancer and prevented its appearance. Without cancer we probably would have continued to carelessly expose our bodies to harmful substances and radiation. Such behavior could have resulted in disastrous consequences to our human species with probable sudden high death rates. This outcome may be extended to other animals and also to plants. The appearance of cancer, however, allows us to see the negative consequences of exposure to radiation and those chemicals we came to label as carcinogens. Therefore, cancer explains the 
hasty and premature death seen in patients developing this disease and which can be mediated. Therefore, cancer appearance could be qualified, in this evolutionary frame, as the alarm calling to save our human species.

\section{HOW TELOMERASE AND PARP INHIBITION FAILED TO CURE CANCER}

The aim of this work is not to review the literature on telomerase and PARP inhibition and inhibitors. Nor is it the objective of this work to make a biased selection of anti-cancer drugs in order to prove a particular point. But the aim of this work is to attract attention to the resistance phenomenon also seen with other anti-cancer drugs and other therapeutic modalities such as radiation therapy.

Telomerase and PARP are chosen as examples of two types of molecules which are reactivated and over-expressed, respectively. The usage of telomerase inhibitors is older and more data are thus available on the subject. PARP inhibition was however still in its clinical trial phase when resistance cases have been reported. Registering such resistance phenomena had, however, helped researchers learn more not only on the mechanisms behind this resistance, but also on the process of cancer development.

\section{Telomerase Inhibition}

Telomeres are repetitive nucleotide sequences at each chromosome end [49], which are coated by a six-protein complex known as shelterins. These molecules play a protective role mechanism against chromosomal degradation, end-to-end fusions and DNA damage responses [50]. Because the cell's replication machinery is unable to provide a complete replication of chromosome ends, chromosomes shorten, as a result, after each cell division. In most organisms, the main mechanism of telomere length maintenance is assured by the telomerase consisting of a reverse transcriptase catalytic subunit (TERT) and an RNA template subunit (TERC) [51, 52]. A non-telomerase mechanism using a DNA template is also known and called alternative lengthening of telomeres (ALT) [53].

In healthy cells, telomerase activity is mostly limited to embryonic cells, adult germ line cells, and stem cells, but is virtually absent in somatic cells [54], while it is common in the vast majority of cancer cells, offering an important target in anti-cancer drug design [55]. The studies across many tumor types have shown that the vast majority of tumors $(\sim 85 \%)$ express telomerase [56]. The remaining $15 \%$ of tumors either do not maintain telomere length or activate the ALT mechanism [57].

In a relatively recent work by $\mathrm{Hu}$ et al. [58] from De Pinho's lab at MD Anderson, it has been shown that telomerase reactivation promotes tumorigenesis by stabilizing telomeres and alleviating the telomere dysfunction-induced checkpoints, and that telomerase reactivation in spontaneous tumors with unstable genomes promotes aggressive malignant properties. Most importantly though, the genetic extinction of telomerase inhibits cancer growth but also leads to tumor re-emergence through the ALT mechanism, providing evidence that cancer cells adapt by taking secondary routes.

\section{PARP Inhibition}

Poly (ADP) polymerase (PARP) is a polymerase required to repair DNA damage and also plays a role in cell division, differentiation and transformation [59]. 17 members of the PARP super-family have so far been identified in 
humans, with PARP-1 being the major form that has been most studied and accounts for more than $90 \%$ of cellular PARP activity [60].

The main forms of DNA damage are single strand breaks (SSB), double strand breaks (DSB) or replication fork stalling. In these cases, PARP-1 molecules are recruited to regulate the process of auto-parylation. The PAR polymers that are synthesized reseal and repair the damage and, once this is accomplished, they dissociate from the DNA-PARP repair complexes. Small molecules of PARP inhibitors (like BMN-673) cause the trapping of PARP-1 at sites of DNA damage, preventing, thus, the accessibility to the repair site of other repair proteins [61].

While several PARP inhibitors were still in phase 1 and 2 clinical trials as mono- or combination therapies to assess their safety and efficacy [62], three mechanisms of resistance to PARPi therapy have been identified so far. These mechanisms are: (1) up-regulation of $\operatorname{PgP}$ transporter, (2) loss of PARP-1 expression, (3) restoration of the homologous recombination (HR) pathway in BRCA targeted tumors [63, 64].

While Up-regulation of $\operatorname{PgP}$ pumps is a common pharmacological effect that reduces the efficacy of a number of drugs including PARP inhibitors by refluxing the drugs out of the cell, BRCA2 mutant patients have shown resistance to PARP inhibitors by creating a secondary mutation in the BRCA2 gene that restores the open reading frame, restoring thus its function. Another way of resisting PARP inhibitors has been noted clinically via restoration of the homologous recombination (HR) pathway and is specific to breast cancer susceptibility gene (BRCA)-targeted tumors [63].

In conclusion, knowing how cancer treatment with telomerase and PARP inhibitors gave rise to resistance leads us to ask the why question. Such resistance of cancer cells shows again their remarkable adaptation capacity. More importantly though, this resistance may indicate that these intelligently designed two anti-cancer drugs are probably acting on the symptoms of tumors rather than their cause.

\section{THE FUTURE OF ANTI-CANCER DRUGS AND CANCER THERAPY}

Registering such resistance phenomena to telomerase and PARP inhibition and also to other anti-cancer drugs such as anti-angiogenic drugs and other cancer treatment modalities raises attention to what really causes cancer.

\section{Defining the Cause of Cancer}

In a recent study on colorectal carcinoma (CRC) by Dow et al. [65] from SW Lowe's lab, adenomatous polyposis coli (APC) loss was defined as the initiating and driving event in CRC because APC silencing blocks differentiation and drives hyper-proliferation in the intestine. More importantly, these authors demonstrated that the restoration of APC promotes disease regression in the small and large intestine while re-establishing homeostasis in the intestinal crypt.

In the light of the present work and if APC loss were the cause in CRC development, APC loss should also be the cause in other types of cancers regardless of the nature of the tissue since we are dealing with the same pathology characterized mainly by uncontrolled cell growth. Moreover, APC loss of function mutation is seen in only $80-90 \%$ of the reported CRC cases. Had APC loss been the cause in the CRC development, it would have been lost in $100 \%$ of the CRC cases.

The cause of cancer as inferred to in this work is defined as the initial cellular change 
that makes a normal cell deviate from regulated cell division and engage in deregulated cell division. In other words, it is the initial cellular change; without it, transformation cannot occur. This pathological change initiating cancer should be $100 \%$ present in all transformed cells and $0 \%$ present in their normal counterparts. Here, and for purposes of clarity only, the initial cellular change causing cancer could be seen as a mutated protein found in all cancer cells while its counterpart remains unchanged in healthy cells.

Therefore, there shouldn't be multiple causes for multiple cancers because cancer is the same pathology, showing different symptoms in different tissues having diverse functions. These symptomatic differences of cancer could be related to cancer cells not following universal laws for their division, their high rate of mutation and also their high capacity for adaptation and ability to hijack numerous metabolic pathways.

When the cause of cancer becomes known, then future anti-cancer drugs should target the causing agent of cancer. The drugs targeting the cause of cancer should be able to shrink the initial tumor(s), but, most importantly, should prevent the rising of new cancer cells harboring new mutations. Targeting the cause of cancer is also expected to have much less side effects as compared to those seen with the presently used cytotoxic anti-cancer drugs.

\section{Understanding Senescence}

Since senescence is one important form of protection against cancer, understanding senescence becomes pivotal, along with the deciphering of the interplay between senescence and apoptosis (Fig. 1). Understanding how cells escape senescence when under intense stress is crucial to addressing the cause of cancer. The star players on the field of senescence are the well-known p53-p21 and p16-RB proteins involved in the axis of both replication and stress-induced senescence. Senescence is important to understand because analysis of several types of pre-malignant tumors, most notably benign skin nevi, has revealed the existence of senescent pre-tumorigenic cells [66]. It has been demonstrated that pre-malignant human nevi and colon adenomas contained cells that express senescence markers, including SA-Bgal and DDR signaling; however, senescent cells were markedly diminished in the malignant melanomas and adenocarcinomas that develop from these lesions $[67,68]$.

On the other hand, it is known that the stimuli that bring forth a senescence response all have the potential to initiate or promote carcinogenesis [69]. As stated by Hanahan and Weinberg [70], to form a lethal tumor, cancer cells must acquire a greatly expanded growth potential and ability to proliferate while expressing activated oncogenes. These features are the very ones suppressed by the senescence program when cells senesce. Furthermore, cellular senescence depends critically on two powerful tumor suppressor pathways: the p53 and $\mathrm{pRB} / \mathrm{p} 16 \mathrm{INK} 4 \mathrm{a}$ pathways. Both pathways integrate multiple aspects of cellular physiology to determine and orchestrate cell fate, and organisms in which cells fail to undergo senescence do not live longer; rather they die prematurely of cancer [69].

The functional relevance of spontaneous senescence induction in preventing tumor initiation and progression has been demonstrated in mouse studies [71, 72]. However, the goal of understanding senescence is not to force cancer cells to senesce because the idea of facilitating senescence as a method of 
cancer therapy may result in harmful side effects [73]. The key question here is what prevents cells from senescing when under stress and rather choose to go cancerous? This is different from forcing cells to senesce once they have become cancerous. The concern here is specificity as we cannot force tumor cells to senesce while sparing normal cells from senescing at an accelerated rate. Similarly, forcing cancer cells to enter apoptosis may also result in harmful side effects because of a lack of specificity. Moreover, deciphering the interplay between apoptosis and senescence will offer unprecedented new possibilities not only for designing future anti-cancer drugs but also for understanding other diseases as well such as neurodegenerative diseases.

\section{CANCER IMMUNOTHERAPY: WHAT TO TARGET?}

Cancer immunotherapy emerged as a practical approach for cancer treatment in which the patient's own immune system is enhanced to attack tumors, and the past decades have known significant progress in this field. Immunotherapy strategies include antitumor monoclonal antibodies, adoptive transfer of ex vivo activated $\mathrm{T}$ and natural killer cells, cancer vaccines, and administration of antibodies or recombinant proteins that either co-stimulate immune cells or block immune inhibitory pathways known as immune checkpoints [74, 75]. Targeting the immune regulatory mechanisms, such as blocking the interaction between the programmed cell death (PD)-1 protein and one of its ligands, PD-L1, has led to clinically meaningful results in several malignancies, including the most aggressive form of cancers, i.e. melanoma [76-81].
The concept of cancer immunotherapy has thus provided a fresh perspective as it is not associated with many of the shortcomings of conventional therapies such as chemotherapy, radiotherapy and surgery [82]. However, and because of the fact that the different mechanisms targeted in immunotherapy do not function independently, several challenges rose as a consequence. For instance, early efforts in cancer vaccination did not give reproducible results. This paved the way to search for tumor-associated antigens, hoping that specific targeting of these antigens would lead to successful tumor eradication. Efforts in cytokine therapy were also met with difficulties, such as a high systemic toxicity and a lack of specific lymphocyte activation [82]. Additionally, attempts at alleviating some of the challenges in cancer immunotherapy have led to combinatorial strategies such as chemotherapy and immunotherapy, knowing that neither of these two types of treatment by itself has been sufficient to eradicate the disease [83].

Though durable clinical remissions have been observed with various immunotherapeutic strategies, the percentage of patients who benefited from these interventions has remained too small to justify the general use of such strategies [80, 84]. Hence, the main questions asked here are: (1) what is intended in immunotherapy as a cancer treatment modality, and (2) what to target in cancer immunotherapy?

If the goal is to improve the prognosis and survival rates, we'll then continue to look for that ideal combination that will give us high rates of long clinical responses without severe toxicities. This will entail careful dose titrations in order to define windows of clinical efficacy $[85,86]$. But if the goal is the eradication of 
cancer as well as the prevention of its recurrence, then targeting the cause of cancer will do it. Sure enough, the hidden challenge remains in whether we want to follow the same road as we did with chemotherapy, ending up with treatment combinations, increased toxicity without achieving complete cure, or do we want to take a road never travelled before, target the initial cellular change that sets on transformation and prevent the rising of cancerous cells? Cancer immunotherapy that is following the traditional chemotherapy road translates but into another symptom-based therapy simply because a different metabolic pathway is being targeted, the immune reactions, instead of DNA-repair or telomere-lengthening pathways or any other metabolic pathway that might be targeted for that matter.

Regardless of the challenges still encountered, developing therapies to enhance tumor immunity or circumvent immune suppression remains, however, of profound importance for cancer therapy. In the long run, the development of a prophylactic vaccine that will prevent transformation makes all challenges worth taking. The knowledge gained today will certainly serve tomorrow's goals and cure cancer when the cause of cancer becomes known.

\section{CONCLUSION}

This paper defines the pathology of cancer in its global context and this is important because a problem well-defined is a problem half-solved. Moreover, it offers a lead for future cancer research and therapy.

This study shows that natural forces governing evolution may not have selected for cancer. Cancer arises when some cells take a detour from regulated cell division seeking immortality, and heterogeneity seen in tumors could be indicative of cancer cells not following universal laws governing cell division.

Cancer hallmarks may be grouped in three stages with the first stage being the most important where the reprogramming events take place in a discreet manner. The resistance phenomenon seen with the current anti-cancer drugs may indicate that these drugs are not acting on the cause of tumors. Future research should merge toward detecting the initial cellular change that is behind the onset of transformation. While immunotherapy holds great prospects for future cancer cures and prevention, the molecular cause of cancer should be addressed. Modeling transformation as being initiated by a single cellular change will be the subject of a future investigation.

Moreover, caring for our cancer patients might require more than chemical molecules because these patients could be depressed following the diagnosis of their disease. Therefore, it might be reasonable to dispense simultaneously a psychotherapeutic treatment. The aim is to make cancer patients participate in their healing process, strengthen them and help them erase the memory of cancer.

Finally, global changes are needed in order to minimize and control the usage of harmful substances known for their contribution in cancer development.

\section{ACKNOWLEDGMENTS}

No funding or sponsorship was received for this study or publication of this article. All authors had full access to all of the data in this study and take complete responsibility for the integrity of the data and accuracy of the data 
analysis. All named authors meet the International Committee of Medical Journal Editors (ICMJE) criteria for authorship for this manuscript, take responsibility for the integrity of the work as a whole, and have given final approval for the version to be published.

Disclosures. A. Adjiri has nothing to disclose.

Compliance with Ethics Guidelines. This article is based on previously conducted studies and does not involve any new studies of human or animal subjects performed by any of the authors.

Open Access. This article is distributed under the terms of the Creative Commons Attribution-NonCommercial 4.0 International License (http://creativecommons.org/licenses/ by-nc/4.0/), which permits any noncommercial use, distribution, and reproduction in any medium, provided you give appropriate credit to the original author(s) and the source, provide a link to the Creative Commons license, and indicate if changes were made.

\section{REFERENCES}

1. Kim JJ, Tannock IF. Repopulation of cancer cells during therapy: an important cause of treatment failure. Nat Rev Cancer. 2005;5:516-25.

2. Bernier J, Hall EJ, Giaccia A. Radiation oncology: a century of achievements. Nat Rev Cancer. 2004;4:737-47.

3. Harada H. How can we overcome tumor hypoxia in radiation therapy? J Radiat Res. 2011;52:545-56.

4. Huang L, Snyder AR, Morgan WF. Radiation-induced genomic instability and its implications for radiation carcinogenesis. Oncogene. 2003;22:5848-54.

5. Wild-Bode C, Weller M, Rimner A, Dichgans J, Wick W. Sublethal irradiation promotes migration and invasiveness of glioma cells: implications for radiotherapy of human glio-blastoma. Cancer Res. 2001;61:2744-50.

6. Karger CP, Jäkel O. Current status and new developments in ion therapy. Strahlenther Onkol. 2007;183:295-300.

7. Kalia M. Biomarkers for personalized oncology: recent advances and future challenges. Metabolism. 2015;64(3 Suppl 1):S16-21.

8. Harrabi S, Combs SE, Brons S, Haberer T, Debus J, Weber KJ. Temozolomide in combination with carbon ion or photon irradiation in glioblastoma multiforme cell lines-does scheduling matter? Int J Radiat Biol. 2013;89:692-7.

9. Formenti SC, Demaria S. Combining radiotherapy and cancer immunotherapy: a paradigm shift. J Natl Cancer Inst. 2013;105:256-65.

10. Vogelstein B, Kinzler KW. Cancer genes and the pathways they control. Nat Med. 2004;10:789-99.

11. Foulds L. Neoplastic development, vol. 1. London: Academic Press; 1969.

12. Weinstein IB. Carcinogenesis as a multistage process-experimental evidence. In: Bartsch $\mathrm{H}$, Armstong B, editors. Host factors in human carcinogenesis (IARC Scientific Publications No. 39). Lyon: IARC Press; 1982. p. 9-25.

13. Nordling CO. BR J Cancer. 1953;7:68-72.

14. Tomasetti C, Marchionni L, Nowak MA, Parmigiani G, Vogelstein B. Only three driver gene mutations are required for the development of lung and colorectal cancers. PNAS. 2015;112:118-23.

15. Hanahan D, Weinberg RA. Hallmarks of cancer: the next generation. Cell. 2011;144(5):646-74.

16. Li Yinghui, Tergaonkar Vinay. Noncanonical functions of telomerase: implications in telomerase-targeted cancer therapies. Cancer Res. 2014;74:1639-44.

17. Gambhir SS. Molecular imaging of cancer with positron emission tomography. Nat Rev Cancer. 2002;2:683-93.

18. Dang CV. Links between metabolism and cancer. Genes Dev. 2012;26:877-90.

19. Al-Husein B, Abdalla M, Trepte M, DeRemer DL, Somanath PR. Anti-angiogenic therapy for cancer: an update. Pharmacotherapy. 2012;32:1095-111. 
20. Colotta F, Allavena P, Sica A, Garlanda C, Mantovani A. Cancer-related inflammation, the seventh hallmark of cancer: links to genetic instability. Carcinogenesis. 2009;30:1073-81.

21. Peebles KA, Lee JM, Mao JT, et al. Inflammation and lung carcinogenesis: applying findings in prevention and treatment. Expert Rev Anti-cancer Ther. 2007;7:1405-21.

22. Smith JR, Pereira-Smith OM. Replicative senescence: implications for in vivo aging and tumor suppression. Science. 1996;273:63-7.

23. Campisi J, di Fagagna FA. Cellular senescence: when bad things happen to good cells. Nat Rev Mol Cell Biol. 2007;8:729-40.

24. Campisi J. Senescent cells, tumor suppression, and organismal aging: good citizens, bad neighbors. Cell. 2005;120:513-22.

25. Kuilman T, Michaloglou C, Mooi WJ, Peeper DS. The essence of senescence. Genes Dev. 2010;24:2463-79.

26. Sherr CJ, Roberts JM. CDK inhibitors: positive and negative regulators of G1-phase progression. Genes Dev. 1999;13:1501-12.

27. Gil J, Peters G. Regulation of the INK4b-ARF-INK4a tumour suppressor locus: all for one or one for all. Nat Rev Mol Cell Biol. 2006;7:667-77.

28. Bennett DC. Human melanocyte senescence and melanoma susceptibility genes. Oncogene. 2003;22:3063-9.

29. Ruas M, Peters G. The p16INK4a/CDKN2A tumor suppressor and its relatives. Biochim Biophys Acta. 1998;1378:F115-77.

30. Bartkova J, Zemanova M, Bartek J. Expression of CDK7/CAK in normal and tumor cells of diverse histogenesis, cell-cycle position and differentiation. Int J Cancer. 1996;66:732-7.

31. Walker GJ, Flores JF, Glendening JM, Lin AH, Markl ID, Fountain JW. Virtually $100 \%$ of melanoma cell lines harbor alterations at the DNA level within CDKN2A, CDKN2B, or one of their downstream targets. Genes Chromosomes Cancer. 1998;2:157-63.

32. Gosselin $\mathrm{K}$, Martien $\mathrm{S}$, Pourtier $\mathrm{A}$, et al. Senescence-associated oxidative DNA damage promotes the generation of neoplastic cells. Cancer Res. 2009;69:7917-25.

33. Campisi J. Cancer, aging and cellular senescence. In Vivo. 2000;14:183-8.
34. Kerr JFR, Wyllie AH, Currie AR. Apoptosis: a basic biological phenomenon with wide-ranging implications in tissue kinetics. $\mathrm{Br} \mathrm{J}$ Cancer. 1972;26:239-57.

35. Baehrecke EH. How death shapes life during development. Nat Rev Mol Cell Biol. 2002;3:779-87.

36. Fuchs Y, Steller H. Programmed cell death in animal development and disease. Cell. 2011;147:742-58.

37. Wong RSY. Apoptosis in cancer: from pathogenesis to treatment. J Exp Clin Cancer Res. 2011;30:87.

38. Bai L, Zhu WG. p53: structure, function and therapeutic applications. J Cancer Mol. 2006;2:141-53.

39. Avery-Kiejda KA, Bowden NA, Croft AJ, et al. p53 in human melanoma fails to regulate target genes associated with apoptosis and the cell cycle and may contribute to proliferation. BMC Cancer. 2011;11:203.

40. Weinert T, Lydall D. Cell cycle checkpoints, genetic instability and cancer. Semin Cancer Biol. 1993;4:129-40.

41. Liu G, Parant JM, Lang G, et al. Chromosome stability, in the absence of apoptosis, is critical for suppression of tumorigenesis in Trp53 mutant mice. Nat Genet. 2004;36:63-8.

42. Lu M, Breyssens H, Salter V, et al. Restoring p53 function in human melanoma cells by inhibiting MDM2 and cyclin B1/CDK1-phosphorylated nuclear iASPP. Cancer Cell. 2013;23:618-33.

43. Mercer WE, Shields MT, Amin M, Sauve GJ, Appella E, Romano JW, Ullrich SJ. Negative growth regulation in a glioblastoma tumor cell line that conditionally expresses human wild-type p53. Proc Natl Acad Sci USA. 1990;87:6166-70.

44. Scheffner M, Werness BA, Huibregtse JM, Levine AJ, Howley PM. The E6 oncoprotein encoded by human papillomavirus types 16 and 18 promotes the degradation of p53. Cell. 1990;63:1129-36.

45. Serrano M, Lin AW, McCurrach ME, Beach D, Lowe SW. Oncogenic ras provokes premature cell senescence associated with accumulation of p53 and p16INK4a. Cell. 1997;88:593-602.

46. Shaw P, Bovey R, Tardy S, Sahli R, Sordat B, Costa J. Induction of apoptosis by wild-type p53 in a human colon tumor-derived cell line. Proc Natl Acad Sci USA. 1992;89:4495-9.

47. Yonish-Rouach E, Resnitzky D, Lotem J, Sachs L, Kimchi A, Oren M. Wild-type p53 induces apoptosis of myeloid leukaemic cells that is inhibited by interleukin-6. Nature. 1991;352:345-7. 
48. Cahill DP, Kinzler KW, Vogelstein B, Lengauer C. Genetic instability and Darwinian selection in tumours. Trends Cell Biol. 1999;9:M57-60.

49. Blackburn EH. Structure and function of telomeres. Nature. 1991;350:569-73.

50. Martínez P, Blasco MA. Telomeric and extra-telomeric roles for telomerase and the telomere-binding proteins. Nat Rev Cancer. 2011;11:161-76.

51. Feng J, Funk WD, Wang SS, et al. The RNA component of human telomerase. Science. 1995;269:1236-41.

52. Nakamura TM, Morin GB, Chapman KB, et al. Telomerase catalytic subunit homologs from fission yeast and human. Science. 1997;277:955-9.

53. Bryan TM, Reddel RR. Telomere dynamics and telomerase activity in in vitro immortalised human cells. Eur J Cancer. 1997;33:767-73.

54. Broccoli D, Young JW, de Lange T. Telomerase activity in normal and malignant hematopoietic cells. Proc Natl Acad Sci USA. 1995;92:9082-6.

55. Wu KJ, Grandori C, Amacker $\mathrm{M}$, et al. Direct activation of TERT transcription by c-MYC. Nat Genet. 1999;21:220-4.

56. Shay JW, Bacchetti S. A survey of telomerase activity in human cancer. Eur J Cancer. 1997;33:787-91.

57. Bryan TM, Englezou A, Gupta J, Bacchetti S, et al. Telomere elongation in immortal human cells without detectable telomerase activity. EMBO J. $1995 ; 14: 4240-8$.

58. Hu J, Hwang SS, Liesa M, et al. Antitelomerase therapy provokes ALT and mitochondrial adaptive mechanisms in cancer. Cell. 2012;148:651-63.

59. de Murcia G, Menissier-de Murcia J, Schreiber V. Poly (ADP-ribose) polymerase: molecular biological aspects. BioEssays. 1991;13:455-62.

60. Dantzer F, Schreiber V, Niedergang C, et al. Involvement of poly (ADPribose) polymerase in base excision repair. Biochimie. 1999;81:69-75.

61. Murai J, Huang SY, Renaud A, et al. Stereo specific PARP trapping by BMN 673 and comparison with olaparib and rucaparib. Mol Cancer Ther. 2014;13:433-43.

62. Fong PC, Boss DS, Yap TA, et al. Inhibition of poly(ADP-ribose) polymerase in tumors from BRCA mutation carriers. N Engl J Med. 2009;361:123-34.
63. Lord CJ, Ashworth A. Mechanisms of resistance to therapies targeting BRCA-mutant cancers. Nat Med. 2013;19:1381-8.

64. Bouwman P, Jonkers J. Molecular pathways: how can BRCA-mutated tumors become resistant to PARP inhibitors? Clin Cancer Res. 2014;20:540-7.

65. Dow LE, O'Rourke KP, Simon J, Tschaharganeh DF, van Es JH, Clevers $\mathrm{H}$, Lowe SW. APC restoration promotes cellular differentiation and reestablishes crypt homeostasis in colorectal cancer. Cell. 2015;161:1539-52.

66. Larsson LG. Oncogene- and tumor suppressor gene-mediated suppression of cellular senescence. Semin Cancer Biol. 2011;21:367-76.

67. Bartkova J, Horejsí Z, Koed K, et al. DNA damage response as a candidate anti-cancer barrier in early human tumorigenesis. Nature. 2005;434:864-70.

68. Michaloglou C, Vredeveld LC, Soengas MS, et al. BRAFE600-associated senescence-like cell cycle arrest of human naevi. Nature. 2005;436:720-4.

69. Rodier F, Campisi J. Four faces of cellular senescence. J Cell Biol. 2011;192:547-56.

70. Hanahan D, Weinberg RA. The hallmarks of cancer. Cell. 2000;100:57-70.

71. Sun P, Yoshizuka N, New L, Moser BA, Li Y, Liao R, et al. PRAK is essential for ras-induced senescence and tumor suppression. Cell. 2007;128:295-308.

72. Lin $\mathrm{HK}$, Chen Z, Wang G, et al. Skp2 targeting suppresses tumorigenesis by Arf-p53-independent cellular senescence. Nature. 2010;464:374-9.

73. Ohtani N, Hara E. Roles and mechanisms of cellular senescence in regulation of tissue homeostasis. Cancer Sci. 2013;104:525-30.

74. Topalian SL, Weiner GJ, Pardoll DM. Cancer immunotherapy comes of age. J Clin Oncol. 2011;29:4828-36.

75. Butt AQ, Mills KHG. Immunosuppressive networks and checkpoints controlling antitumor immunity and their blockade in the development of cancer immunotherapeutics and vaccines. Oncogene. 2014;33:4623-31.

76. Topalian SL, Hodi FS, Brahmer JR, et al. Safety, activity, and immune correlates of anti-PD-1 antibody in cancer. $\mathrm{N}$ Engl $\mathrm{J}$ Med. 2012;366:2443-54. 
77. Topalian SL, Sznol M, Mcdermott DF, et al. Survival, durable tumor remission, and long-term safety in patients with advanced melanoma receiving nivolumab. J Clin Oncol. 2014;32:1020-30.

78. Hamid O, Robert C, Daud A, et al. Safety and tumor responses with lambrolizumab (anti- PD-1) in melanoma. N Engl J Med. 2013;369:134-44.

79. Ansell SM, Lesokhin AM, Borrello I, et al. PD-1 blockade with nivolumab in relapsed or refractory Hodgkin's lymphoma. N Engl J Med. 2015;372:311-9.

80. Moreno BH, Ribas A. Anti-programmed cell death protein-1/ligand-1 therapy in different cancers. Br J Cancer. 2015;112:1421-7.

81. Philips GK, Atkins M. Therapeutic uses of anti-PD-1 and anti-PD-L1 Antibodies. Int Immunol. 2014;27(1):39-46.

82. Chaudhuri D, Suriano R, Mittelman A, Tiwari RK. Targeting the immune system in cancer. Curr Pharm Biotechnol. 2009;10:166-84.
83. Baxevanis CN, Perez SA, Papamichail M. Combinatorial treatments including vaccines, chemotherapy and monoclonal antibodies for cancer therapy. Cancer Immunol Immunother. 2009;58:317-24.

84. Azijli K, Stelloo E, Peters GJ, Van Den Eertwegh AJ. New developments in the treatment of metastatic melanoma: immune checkpoint inhibitors and targeted therapies. Anti-cancer Res. 2014;34:1493-506.

85. Okazaki T, Okazaki IM, Wang J, et al. PD-1 and LAG-3 inhibitory co-receptors act synergistically to prevent autoimmunity in mice. J Exp Med. 2011;208:395-407.

86. Humphrey R, Brockway-Lunardi L, Bonk D, et al. Opportunities and challenges in the development of experimental drug combinations for cancer. J Natl Cancer Inst. 2011;103:1222-6. 